\title{
Transição de fases de zeólita do tipo Faujasita para Sodalita via tratamento térmico
}

\section{Transition phases of zeolite Faujasite type to Sodalite by thermal treatment}

\author{
Katia K. Kaminishikawahara'; Laura J. Pereira²; Éder C. F. de Souza'; Maria Elena Payret Arrúa4; \\ Sandra Regina Masetto Antunes ${ }^{5}$; André V. C. de Andrade ${ }^{6}$; Helton J. Alves ${ }^{7}$ Augusto C. Antunes ${ }^{8}$
}

\section{Resumo}

As zeólitas podem ser usadas em diversas funções como catalisadores (produção de biocombustíveis) e peneiras moleculares (tratamento de áreas contaminadas). Este trabalho tem como objetivo caracterizar as zeólitas obtidas na transição de uma zeólita semelhante à Faujasita para Sodalita, quando submetidos a diferentes tempos de tratamentos térmicos. As zeólitas sintetizadas foram caracterizadas por difração de raios $\mathrm{X}$ onde se identificou as fases cristalinas semelhante a Faujasita, Sodalita, $\mathrm{SiO}_{2}$ e a presença de material amorfo. O tratamento térmico de 4 horas produziu zeólitas com estrutura cristalina semelhante à estrutura cristalina da Faujasita, apresentando sítios básicos, área superficial de $552,7 \mathrm{~m}^{2} \mathrm{~g}^{-1} \mathrm{e}$ volume de poros de $0,3391 \mathrm{~cm}^{3} \mathrm{~g}^{-1}$. Com o aumento do tempo do tratamento térmico houve transição para a fase Sodalita, contendo 0,277 mmol g-1 de sítios básicos ativos, com volume de poros de $0,0651 \mathrm{~cm}^{3}$ $\mathrm{g}^{-1}$ e área superficial $11,38 \mathrm{~m}^{2} \mathrm{~g}^{-1}$. Pelo Método de Rietveld foi identificada e quantificada a presença de Sodalita e Hidrossodalita nas amostras com tempo de reação de 24 e $30 \mathrm{~h}$.

Palavras-chave: Zeólitas. Catalisadores. Peneiras moleculares. Faujasita. Sodalita.

\begin{abstract}
The zeolites can have several functions as catalysts (biofuel production) and molecular sieves (treatment of contaminated areas). This study aims to characterize the zeolites obtained in the transition of a Faujasite like zeolite into a Sodalite, when submitted to different thermal treatment times. The synthesized zeolites were characterized by X-ray diffraction where the crystalline phases were identified: Faujasite, Sodalite, $\mathrm{SiO}_{2}$ and amorphous material. The 4 hours heat treatment produces zeolite crystal structure similar to Faujasite, having basic sites, surface area of $552.7 \mathrm{~m}^{2} \mathrm{~g}^{-1}$, and pore volume of $0.3391 \mathrm{~cm}^{3} \mathrm{~g}^{-1}$. With increasing time of heat treatment was observed the transition to the Sodalite phase witch containing $0.277 \mathrm{mmol} \mathrm{g}^{-1}$ of basic active sites with surface area of $11.38 \mathrm{~m}^{2} \mathrm{~g}^{-1}$ and pore volume of $0.0651 \mathrm{~cm}^{3}$ $\mathrm{g}^{-1}$. By the Rietveld method was identified and quantified the presence of Sodalite and Hidrossodalite in samples with 24 and 30 hours of reaction times.
\end{abstract}

Keywords: Zeolites. Catalysts. Molecular Sieves. Faujasite. Sodalite.

\footnotetext{
${ }^{1}$ Aluna de mestrado do Programa de Mestrado em Bioenergia, Universidade Estadual de Ponta Grossa: kkaori146@gmail.com

${ }^{2}$ Aluna de iniciação científica do Departamento de Engenharia de Materiais, Universidade Estadual de Ponta Grossa: laurapereira80@ yahoo.com.br

${ }^{3}$ Pós-doutorando do Departamento de Química, Universidade Estadual de Ponta Grossa: souza.eder@gmail.com

${ }^{4}$ Docente do Departamento de Química da Universidade Estadual de Ponta Grossa: elenapayret@gmail.com

${ }^{5}$ Docente do Departamento de Química da Universidade Estadual de Ponta Grossa: sr-antunes@bol.com.br

${ }^{6}$ Docente do Departamento de Física da Universidade Estadual de Ponta Grossa: avcandrade@gmail.com

${ }^{7}$ Docente do Setor Palotina da Universidade Federal do Paraná: helton.alves@ufpr.br

${ }^{8}$ Docente do Departamento de Química da Universidade Estadual de Ponta Grossa: augusto.celso@pq.cnpq.br.
} 


\section{Introdução}

As zeólitas são classificadas como aluminossilicatos cristalinos e hidratados, constituídos por metais alcalinos ou alcalinos terrosos. Podem ser estruturados por unidades de óxido de alumínio ou óxido de silício (BRECK, 1974, GRECCO; RANGEL; GONZALEZ, 2013; MELO; RIELLA, 2010). São amplamente utilizadas nas mais diversas funções, por exemplo, como catalisadores heterogêneos no processo de produção de gasolina a partir de óleo cru, como peneira molecular na remoção de íons metálicos e tratamento de efluentes (AGUIAR; NOVAES, 2002; VERMEIREN; GILSON, 2009).

A eficiência das zeólitas em desempenhar diversas funções é atribuída principalmente a sua elevada capacidade de adsorção, grandes áreas superficiais, que dependem do tipo de zeólita, possibilidade de criação de sítios ativos e de acordo com a aplicação desejada e variedade e complexidade de rede de canais que permite diferentes tipos de seletividade de forma (LUNA; SCHUCHARDT, 2001; SILVESTRE; VIEIRA; BARRETO, 2012).

Inúmeros estudos estão sendo desenvolvidos utilizando diversos tipos de materiais como fonte de alumínio e silício para a síntese de zeólitas como: caulim, cinza de casca de arroz, cinza volante de carvão, sílica coloidal e aluminato de sódio entre outros (BRAGA; MORGON, 2007; DALAI et al., 2005; GEORGIEV et al., 2013; GRECCO; RANGEL; GONZALEZ, 2013; OJHA; PRADHAN; SAMANTA, 2004).

A Faujasita é amplamente utilizada na indústria como catalisador devido a sua elevada área superficial, volume de poros e a Sodalita em geral como peneira molecular por apresentar uma grande capacidade de troca catiônica (ALMEIDA; MARTINS; CARDOSO, 2010; BALKUS; LY, 1991; PAZ; ANGÉLICA; NEVES, 2010; SANTANA et al., 2012).
A fim de produzir uma zeólita de maior cristalinidade e pureza, Hildebrando et al. (2012), utilizaram rejeito de caulim obtendo um produto cristalino com estrutura do tipo Faujasita com baixa cristalinidade e elevada quantidade de material amorfo. A fase concorrente na síntese foi a Chabazita e ao fixar parâmetros quanto à quantidade dos reagentes e o aumento apenas do tempo de tratamento térmico, houve a indução da formação de outras fases zeolíticas e diminuição da fase Faujasita. Resultados semelhantes foram observados nos trabalhos de Fernandes-Machado e Miotto-Bigatão (2007) e Fungaro e Izidoro (2006), que visavam obter com a síntese materiais de maior pureza e cristalinidade.

Devido à variedade de funções que as zeólitas desempenham e de materiais que podem ser utilizados para a sua síntese, estudos foram realizados para a produção de um material zeolítico, com maior grau de pureza, de dimensões médias de cristalito e reduzida presença de fases secundárias. E a partir desses experimentos foi observada a transição da zeólita com estrutura cristalina semelhante à Faujasita para a Sodalita.

O objetivo deste trabalho foi o preparo de zeólitas e caracterização da transição de zeólita com estrutura semelhante à Faujasita para uma zeólita com estrutura Sodalita, por meio de tratamento térmico em diferentes tempos. As propriedades físico-químicas das zeólitas obtidas foram avaliadas.

\section{Materiais e Métodos}

Para a síntese das zeólitas foi utilizado uma autoclave constituída por uma camisa metálica no qual foi inserido um cilindro de teflon.

Os reagentes usados foram: aluminato de sódio $\left(\mathrm{NaAlO}_{2}\right)$ da empresa NALCO (Pomerode - SC), Sílica Coloidal SICOL 40 fornecido pela empresa UNAPROSIL (Rio de Janeiro - RJ), hidróxido de sódio micropérolas P.A. $100 \%$ da marca SYNTH e 
água ultrapura (Milli-Q).

A metodologia foi desenvolvida pelo grupo de pesquisa, na qual o $\mathrm{NaAlO}_{2}$ foi homogeneizado na solução aquosa de hidróxido de sódio e posteriormente adicionou-se a esta solução a sílica coloidal, obtendo-se um gel.

O sistema ficou em agitação por alguns minutos e depois foi inserido no teflon e este na camisa metálica sendo a autoclave submetida à temperatura de $120^{\circ} \mathrm{C}$ por diferentes tempos $(4,8,12,24$ e 30 horas). Terminado o tratamento térmico as zeólitas foram filtradas e secas em estufa à temperatura de $60^{\circ} \mathrm{C}$ por 4 horas.

Os materiais zeolíticos sintetizados foram caracterizados por difração de raios X (DRX), utilizando a técnica do pó, com o difratômetro da marca Shimadzu, modelo XRD-6000 operando com tubo de cobre a $40 \mathrm{kV}$ e $30 \mathrm{~mA}$, obtendo-se um comprimento de onda característico de $0,15406 \mathrm{~nm}$, com a varredura para levantamento dos dados de $3^{\circ}$ a $100^{\circ}(2 \theta)$ e passo de $0,02^{\circ}$.

As amostras nas quais foram feitas a análise qualitativa de identificação de fases cristalinas foram submetidas ao modo de varredura contínua com velocidade igual a $2 \% \mathrm{~min}$. E as analisadas pelo Método de Rietveld foram realizadas com o modo de varredura de tempo fixo, com passos de $0,02^{\circ}$ e tempo por passo igual a $10 \mathrm{~s}$. Foram utilizadas fendas de divergência e espalhamento iguais a $1,0^{\circ}$ e fenda de recepção de $0,15 \mathrm{~mm}$ em ambos os casos.

Posteriormente, os dados foram tratados com o software OriginPro versão 8.0, no qual foi realizado a identificação das fases presentes utilizando o banco de dados da International Zeolite Association (IZA, 2015).

A determinação do tamanho do cristalito da zeólita Sodalita e com estrutura semelhante a da Faujasita foram realizadas a partir da equação de Scherrer, considerando-se uma distribuição isotrópica das dimensões dos cristais. O valor de $\theta$ para o pico (111) da Faujasita e (110) da Sodalita foram respectivamente, $3,08^{\circ}$ e $6,98^{\circ}$, o valor da constante $\mathrm{K}$ foi de 0,9 admitindo que os cristalitos possuíssem formas esféricas e $\beta$ corresponde a largura a meia altura calculada pelo software OriginPro.

A análise pelo Método de Rietveld foi realizada com o programa GSAS+Expgui (LARSON; VON DREELE, 2000; TOBY, 2001). A função de perfil adotada foi a pseudo-Voigt que incluiu o modelo fenomenológico de Peter Stephens (STEPHENS, 1999) para correção do alargamento de linha anisotrópico, eficiente em casos onde o alargamento da linha de difração não é uma função lisa do espaçamento interplanar. A quantificação das fases presentes nas amostras foi realizada pelo modelo de Howard e Hill (HILL; HOWARD, 1987).

Também foi realizada a análise textural por isoterma de adsorção de nitrogênio para estudo da área superficial específica, volume e características dos poros das zeólitas. Esta análise foi feita no equipamento Surface Area \& Pore Size, modelo NOVA 2000 e da marca Quanta Chrome Instruments, com aquecimento de $200^{\circ} \mathrm{C}$ por 4 horas e emersão em nitrogênio a temperatura de $-273^{\circ} \mathrm{C}$ com acompanhamento pelo software Quantachrome TM Nova Win e tratamento dos dados com o programa OriginPro versão 8.0.

Além disso, as zeólitas foram caracterizadas com a análise qualitativa de basicidade e acidez para determinação dos sítios ativos do material zeolítico utilizando a técnica de titulação de Hammett com os indicadores de amarelo de metilo, azul de timol, vermelho neutro, fenolftaleína, 4-nitroanilina e 2,4-dinitroanilina.

E para a análise de quantificação de sítios básicos, foi realizado um estudo quantitativo de basicidade em que foi utilizada a técnica de titulação de Hammett com uma solução de ácido benzóico de $0,01 \mathrm{~mol} \mathrm{~L}^{-1}$ em metanol e soluções indicadoras representados pelos intervalos denominado de $\mathrm{pK}_{\mathrm{BH}}$, na seguinte ordem: $\mathrm{pK}_{\mathrm{BH}}=3,3$ (amarelo de metilo), $\mathrm{pK}_{\mathrm{BH}}=6,8$ (vermelho neutro), $\mathrm{pK}_{\mathrm{BH}}=8,8$ (azul 
de timol), $\mathrm{pK}_{\mathrm{BH}}=9,8$ (fenolftaleína), $\mathrm{pK}_{\mathrm{BH}}=15$ (2,4-dinitroanilina) e $\mathrm{pK}_{\mathrm{BH}}=18,4$ (4-nitroanilina) (JANKULOVSKA et al., 2011).

\section{Resultados e discussões}

Utilizando a técnica de DRX foi possível a obtenção de informações quanto às fases cristalinas presentes, as dimensões médias do cristalito e a indexação dos picos de difração.

O difratograma da Figura 1 mostra a presença de duas fases, a zeólita com cela unitária semelhante à estrutura cristalina da Faujasita e quartzo $\left(\mathrm{SiO}_{2}\right)$ para a amostra sintetizada em tratamento térmico de 4 horas. Observa-se também um pico alargado na região de $20^{\circ}-40^{\circ}$, referente a uma fase amorfa.

Figura 1 - Difratograma de raios X para a amostra com tratamento térmico de 4 horas, com a indexação das fases presentes.

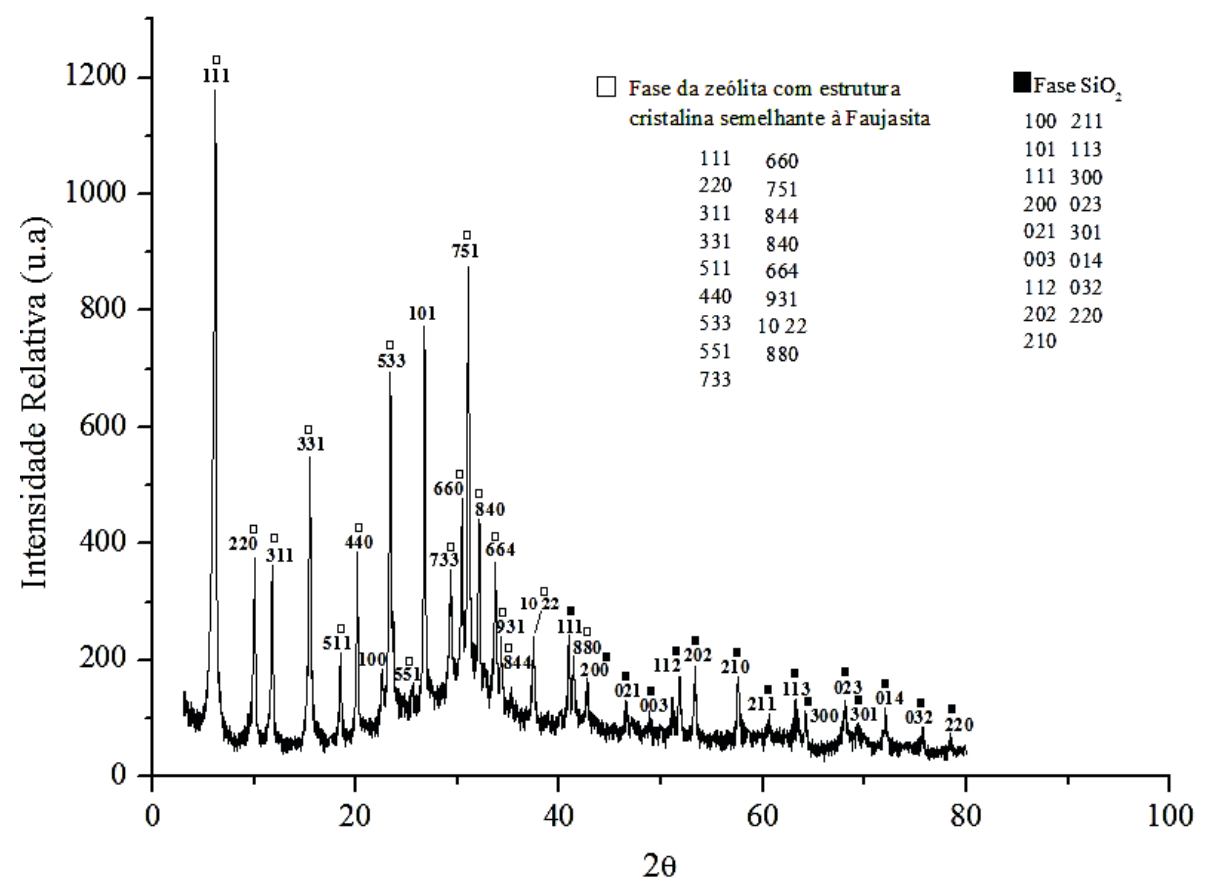

Fonte: Autor.

Com o aumento do tempo de tratamento térmico para 8 horas, houve o aparecimento das fases de Sílica Tetragonal e Sodalita juntamente com a zeólita de estrutura cristalina semelhante a da Faujasita (Figura 2).

Para esta zeólita o tamanho médio do cristalito foi de 21,9 nm enquanto para a Sodalita de 23,5 nm.

Para o tratamento térmico de 12 horas (Figura 3), a zeólita produzida ainda apresentou 3 fases (Sodalita, material zeolítico com estrutura cristalina semelhante à Faujasita e $\mathrm{SiO}_{2}$ ).
Para o tratamento térmico de 24 horas, a fase da zeólita com cela unitária semelhante à Faujasita desapareceu e pode ser observado apenas os picos característicos da Sodalita e de $\mathrm{SiO}_{2}$ (Figura 4).

Para 30 horas de tratamento térmico, foi caracterizado a presença da fase Sodalita e de $\mathrm{SiO}_{2}$ (Figura 5).

A Tabela 1 mostra os resultados obtidos para diferentes tratamentos térmicos em relação à dimensão média do cristalito das fases presentes nos materiais zeolíticos. 
Tabela 1 - Resultados da determinação da dimensão média do cristalito das fases para diferentes tratamentos térmicos.

\begin{tabular}{|c|c|c|}
\hline \multirow[t]{2}{*}{$\begin{array}{c}\text { Tratamento Térmico } \\
\text { (horas) }\end{array}$} & \multicolumn{2}{|c|}{$\begin{array}{c}\text { Dimensões } \\
\text { médias } \\
\text { do cristalito }(\mathbf{n m})\end{array}$} \\
\hline & FAU & SOD \\
\hline 4 & 29,7 & - \\
\hline 8 & 21,9 & 23,5 \\
\hline 12 & 28,9 & 25,6 \\
\hline 24 & - & 33,7 \\
\hline 30 & - & 27,4 \\
\hline
\end{tabular}

Fonte: Autor.

Figura 2 - Difratograma de raios X para a amostra com tratamento de 8 horas com aparecimento das fases Sodalita e Sílica Tetragonal juntamente com a zeólita com cela unitária semelhante à Faujasita.

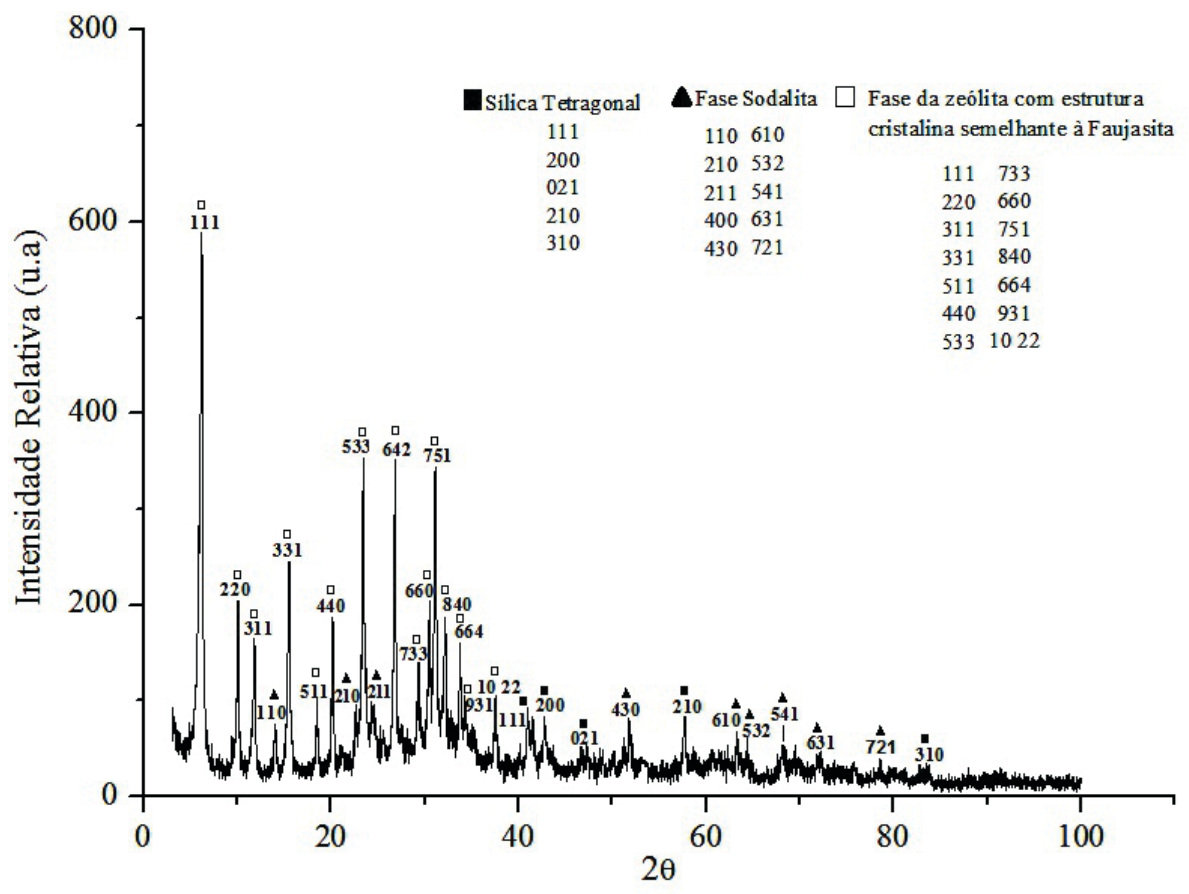

Fonte: Autor. 
Figura 3 - Difratograma do tratamento térmico de 12 horas com presença das fases Sodalita, $\mathrm{SiO}_{2}$ e de material zeolítico com estrutura cristalina semelhante à Faujasita.

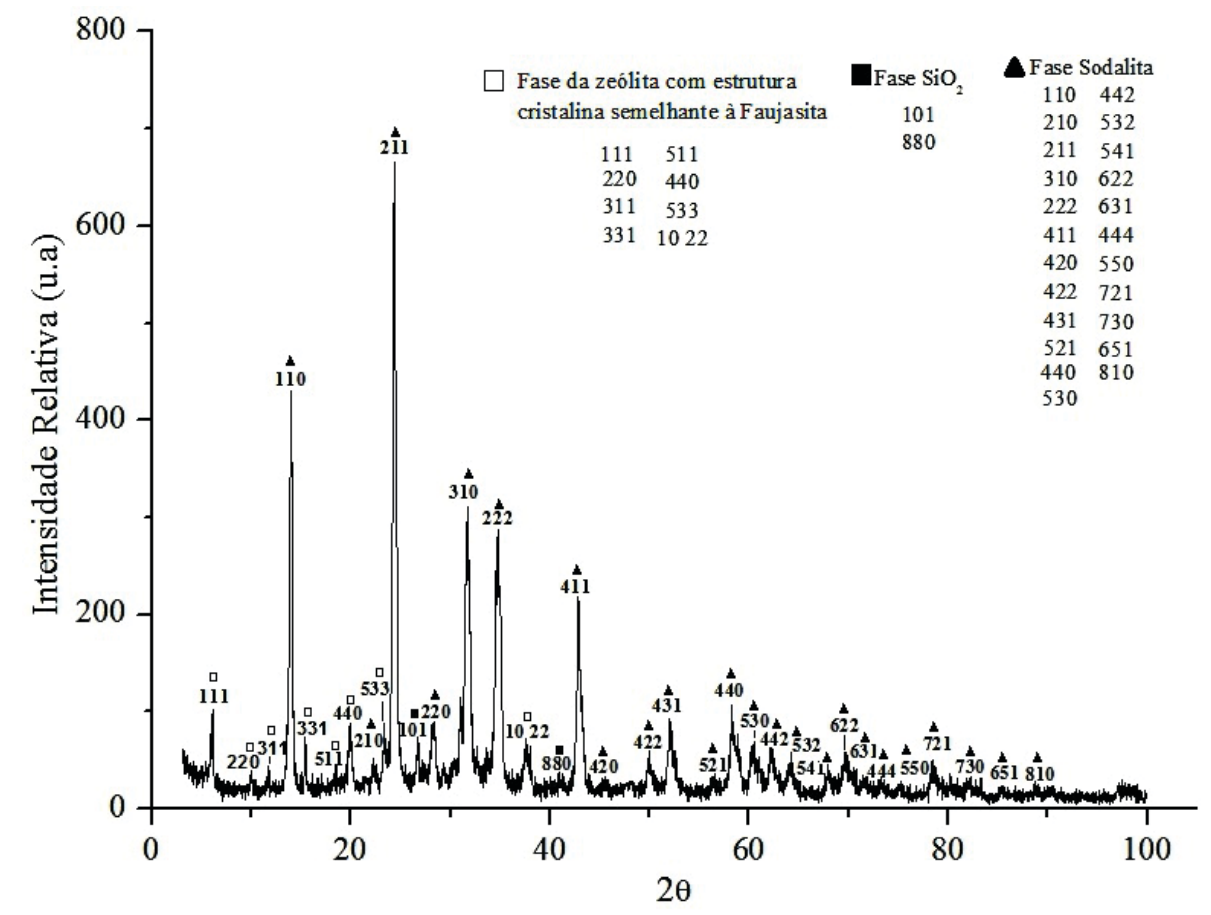

Fonte: Autor.

Conforme a comparação dos tratamentos térmicos formados serão maiores (SIMÕES et al., 2012). A realizados (Tabela 1), quanto maior o tempo de presençadafase Sodalita observadas nos difratogramas da tratamento térmico obtém-se picos de difração de raios Figura 2, Figura 3, Figura 4 e Figura 5 estão condizentes $\mathrm{X}$, mais estreitos, indicando que o tamanho dos cristalitos com os relatados por Santos et al. (2012).

Figura 4 - Difratograma de raios $\mathrm{X}$ obtido para a amostra com tratamento térmico de 24 horas, presença das fases Sodalita e $\mathrm{SiO}_{2}$.

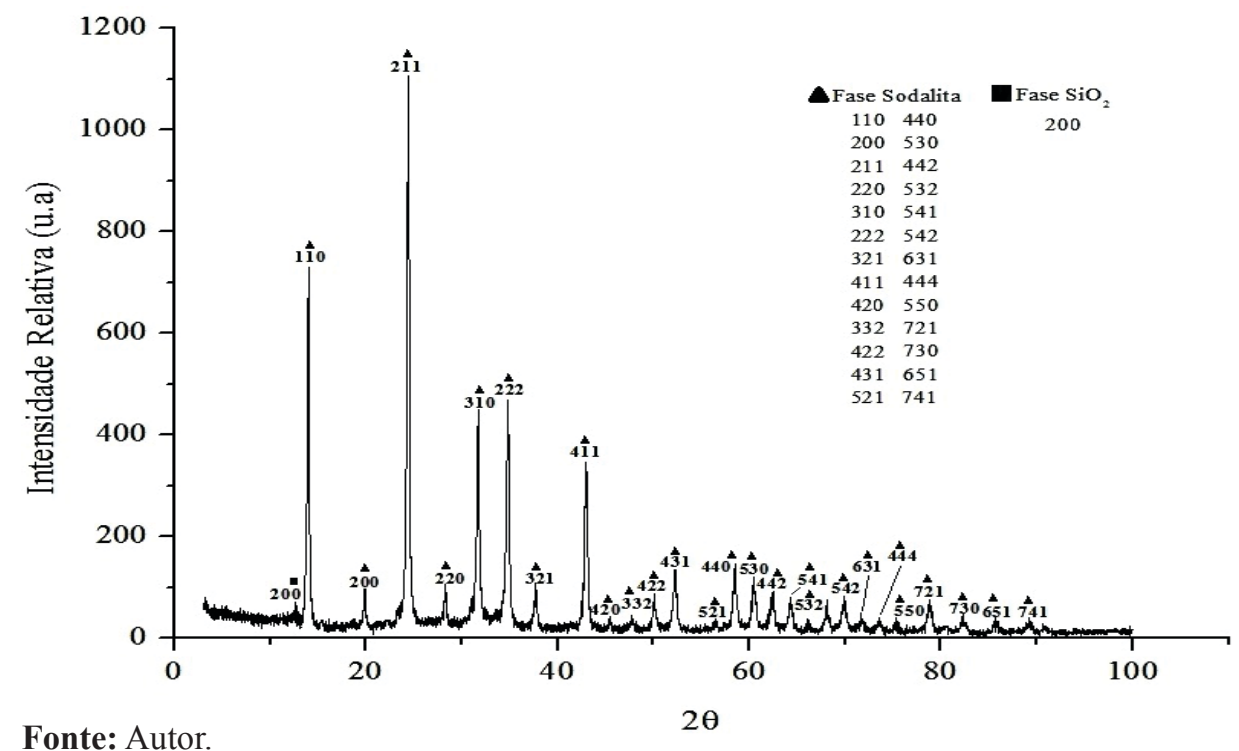

Fonte: Autor. 
Figura 5 - Difratograma de raios X para a amostra com tratamento térmico de 30 horas com dados da indexação das fases presentes.

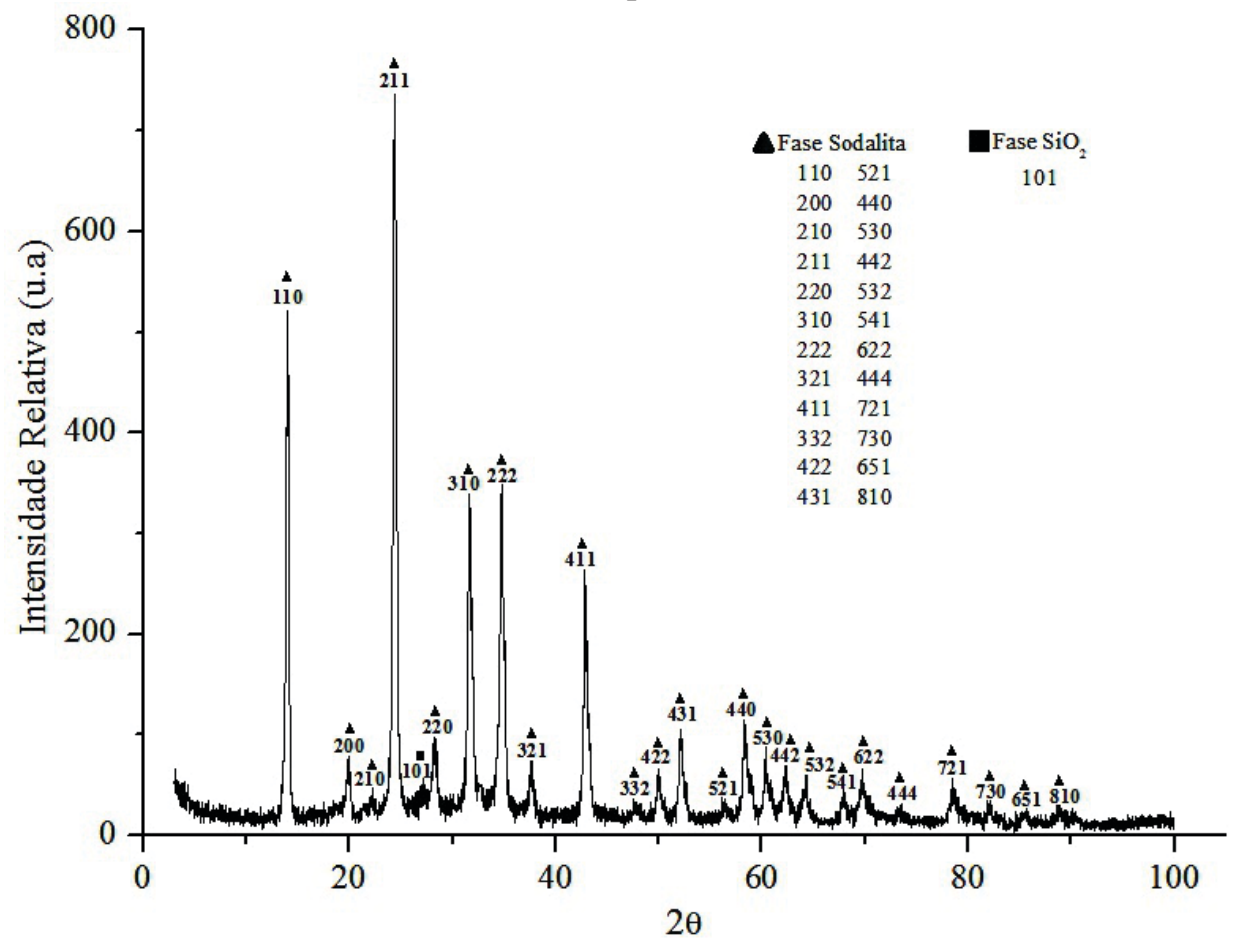

Fonte: Autor.

De acordo com Plotegher e Ribeiro (2009) e Poole, Prijatama e Rice (2000), quanto maior é a temperatura e o tempo de cristalização durante o tratamento térmico, maior a propensão em se formar zeólitas com menores tamanhos de poros, como é o caso da Sodalita que possui uma maior estabilidade nessas condições.

A fim de sintetizar zeólitas do Tipo A e a Faujasita, Fernandes-Machado; Miotto-Bigatão (2007) também relataram o surgimento de uma fase concorrente (Sodalita).

A presença de $\mathrm{SiO}_{2}$ em todos os materiais zeolíticos sintetizados está atrelado possivelmente a resistência dessa fase nos aluminossilicatos e a pouca degradabilidade das fases suportadas por alumínio-silício (FUNGARO; IZIDORO; BRUNO, 2009).

As zeólitas sintetizadas com tratamento térmico de 24 e 30 horas foram caracterizadas pelo Método de Rietveld e houve uma tentativa para o estudo do material zeolítico produzido no tratamento de 4 horas por esta análise. $\mathrm{Na}$ qual se concluiu que a zeólita sintetizada no tratamento de 4 horas apresentava parâmetros de cela unitária semelhante à estrutura cristalina da Faujasita.

Para a Sodalita sintetizada com o tempo de 24 horas, a análise utilizando o Método de Rietveld mostrou que $53 \%$ em quantidade de massa pertenciam à fase Sodalita básica, 46\% a Hidrossodalita (sodalita hidratada) e $1 \%$ dividido entre Cristobalita $\left(\mathrm{SiO}_{2}\right)$ e Óxido de sódio $\left(\mathrm{Na}_{2} \mathrm{O}\right)$ (Figura 6). O índice de discordância (Rwp) para o tratamento térmico de 24 horas foi de $6,92 \%$, fator de qualidade (s) de 1,29 e fator de estrutura, $\mathrm{R}\left(\mathrm{F}^{2}\right)$, indicando o ajuste de $2,44 \%$.

No tratamento de 30 horas a porcentagem em massa de Sodalita básica foi de $37 \%$, a Hidrossodalita de $62 \%$ e para o Superóxido de sódio $\left(\mathrm{NaO}_{2}\right)$ de $1 \%$ (Figura 7). O Rwp calculado foi de $6,92 \%$, o fator de qualidade de 1,30 e o $\mathrm{R}\left(\mathrm{F}^{2}\right)$ de $4,62 \%$. 
Esses resultados evidenciam uma boa qualidade de refinamento, considerado satisfatório e confiável quando alcança baixos índices de discordâncias (LOIOLA et al., 2010). Alguns autores ressaltam que a presença de Hidrossodalita é quase inevitável em processo de síntese de zeólitas (PAZ; ANGÉLICA; NEVES, 2010; SMITH; WINGATE; SILVA, 2008).

A presença de superóxido de sódio para o tratamento mais longo (30 horas) em comparação com o de 24 horas está possivelmente relacionada ao fato de que o óxido de sódio é mais estável que o superóxido, este último formado geralmente em condições de pressão e temperaturas altas (SCHECHTER; SHAKELY, 1957; ZHANG et al., 2012).

Como o tratamento térmico de 30 horas apresentou maior quantidade de Hidrossodalita, esperou-se encontrar mais canais estreitos devido à presença de água no interior dos canais, que poderiam dificultar a passagem de certas moléculas, restringindo possivelmente sua utilização como catalisador na produção de biocombustível (MA et al., 2011; WOOLARD et al., 2002).

$\mathrm{Na}$ técnica de adsorção de gases utilizando o método de BET foi possível caracterizar a área superficial específica, o volume de poros, assim como o estudo das isotermas de adsorção/dessorção que forneceram informações quanto o tipo de poros presentes nas amostras.

O estudo da área superficial externa e dos poros é de extrema importância em reações de catálise e adsorção, pois dependendo da área superficial e do diâmetro dos poros é obtido um maior contato do reagente com o catalisador. E como o tamanho dos poros é definido, fornece ao material seletividade à adsorção de uma espécie, o qual o tamanho da molécula terá que ser da mesma grandeza dos poros ou menor. Entretanto, uma área superficial externa muito elevada pode afetar a seletividade e com isso a eficiência do material (AYODELE; ABBAS; DAUD, 2014; TOSHEVA; VALYECHEV, 2005).

Figura 6 - Gráfico gerado utilizando a análise pelo Método de Rietveld para tratamento térmico de 24 horas.

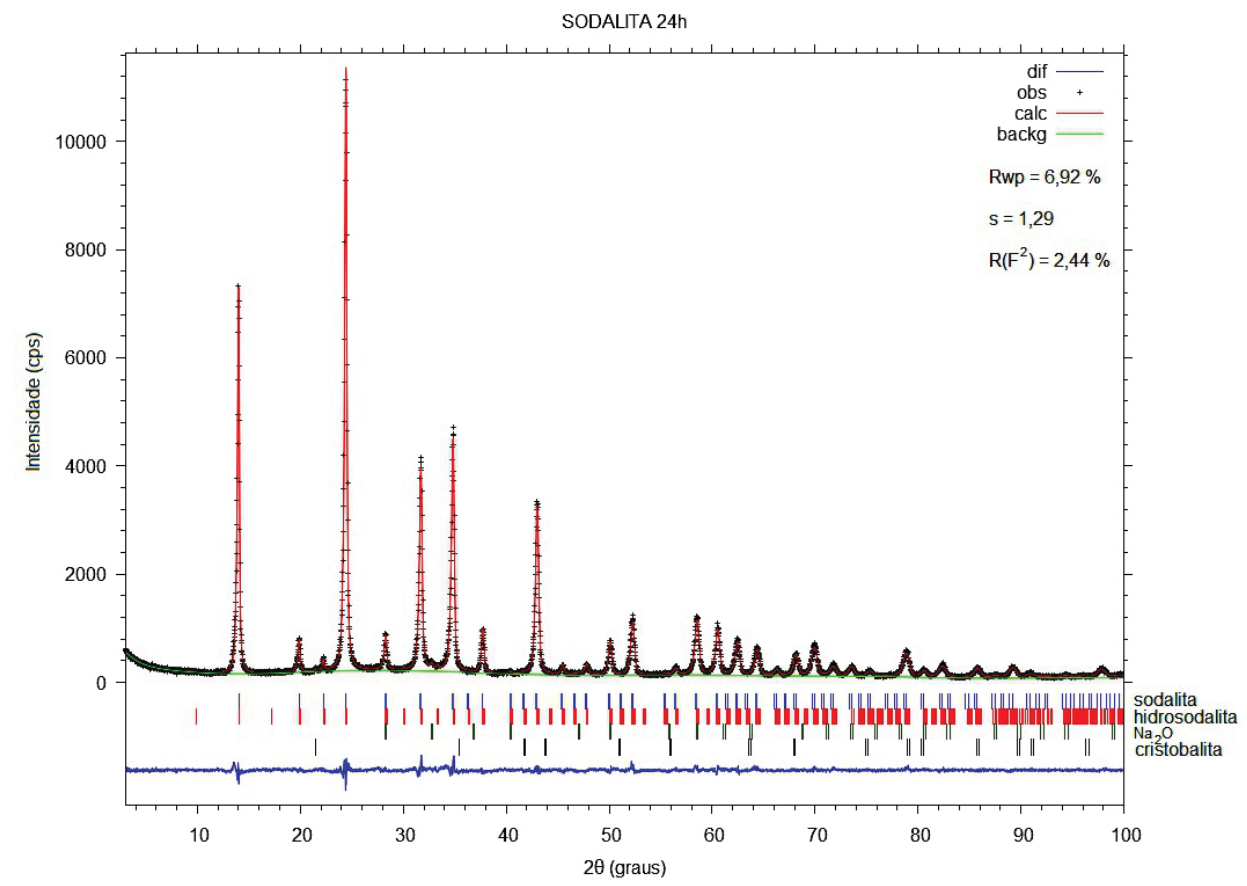

Fonte: Autor. 
Figura 7 - Gráfico gerado pela análise utilizando o Método de Rietveld para as amostras obtidas no tratamento térmico de 30 horas.

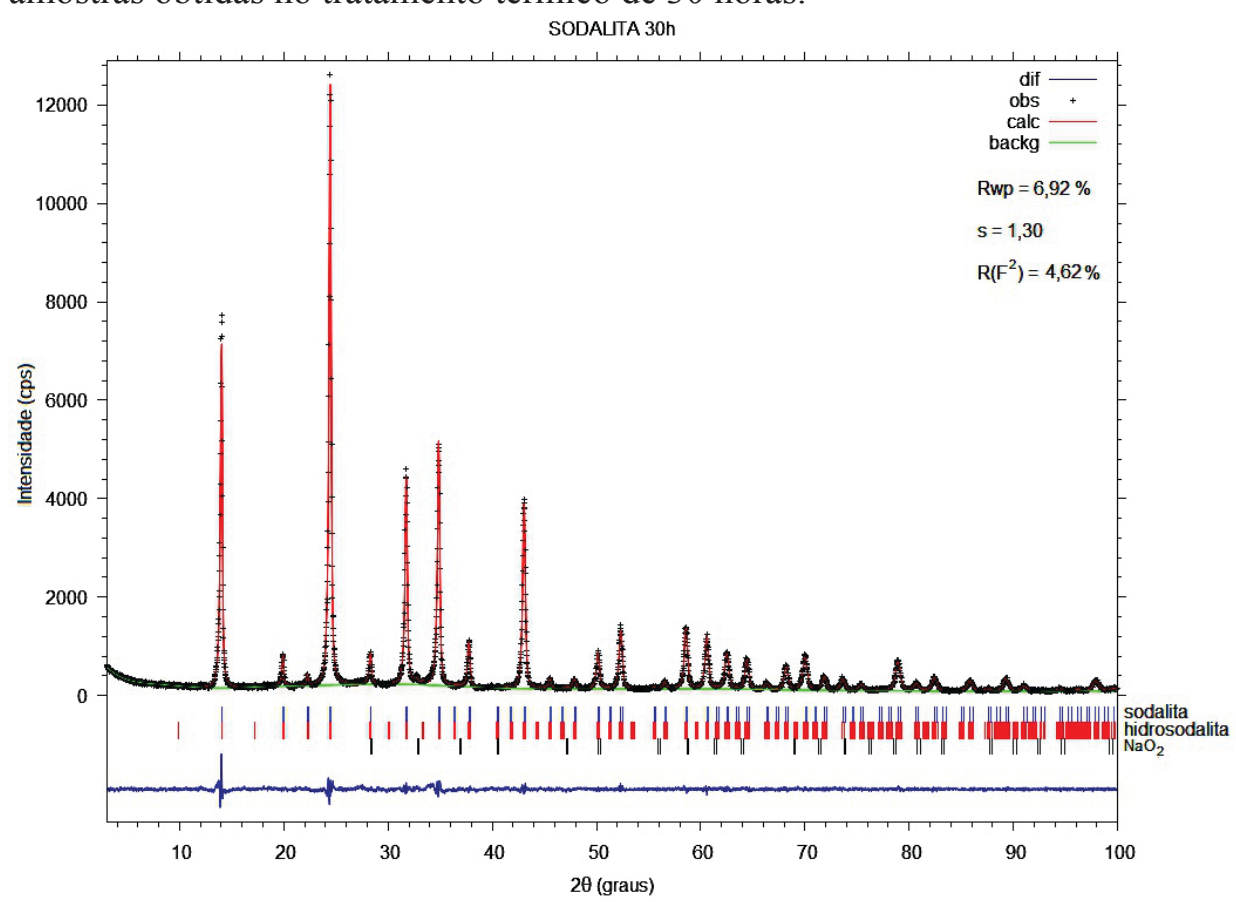

Fonte: Autor.

Para esta técnica de adsorção de gases foram avaliados os produtos obtidos por tratamentos térmicos de 4 horas e 24 horas por apresentarem apenas uma fase zeolítica.

A zeólita com estrutura semelhante à Faujasita sintetizada no tratamento térmico de 4 horas apresentou uma área superficial de $552,7 \mathrm{~m}^{2} \mathrm{~g}^{-1} \mathrm{e}$ volume de poros de $0,3391 \mathrm{~cm}^{3} \mathrm{~g}^{-1}$, superiores ao valores obtidos em zeólita comerciais. Uma zeólita NaX comercial apresenta uma média de área superficial de $332,2 \mathrm{~m}^{2} \mathrm{~g}^{-1}$ e volume de poros de $0,2461 \mathrm{~cm}^{3} \mathrm{~g}^{-1}$ (KUZNICKI et al., 2002, MIOTTO; FERNANDES-MACHADO, 2002; CAOVILLA et al., 2004).
Para a Sodalita produzida no tratamento térmico de 24 horas a área superficial foi de $11,38 \mathrm{~m}^{2} \mathrm{~g}^{-1}$, menor que a relatada na literatura (33-72 $\left.\mathrm{m}^{2} \mathrm{~g}^{-1}\right)$ enquanto o volume de poros de $0,0651 \mathrm{~cm}^{3} \mathrm{~g}^{-1}$ está dentro do intervalo de outros trabalhos sobre o assunto $\left(0,0488-0,12 \mathrm{~cm}^{3} \mathrm{~g}^{-1}\right)$ (HIYOSHI, 2012; FRANUS; WDOWIN; FRANUS, 2014).

A análise das isotermas da zeólita obtida após tratamento térmico de 4 horas apresentou predomínio de microporos (Figura 8) e com 24 horas (Figura 9) de mesoporos. Estes resultados estão de acordo com o relatado nos experimentos de Shanbhag et al. (2009). 
Figura 5 - Isotermas de adsorção para as amostras obtidas pelo tratamento térmico de 4 horas com zeólita com estrutura cristalina semelhante à da Faujasita.

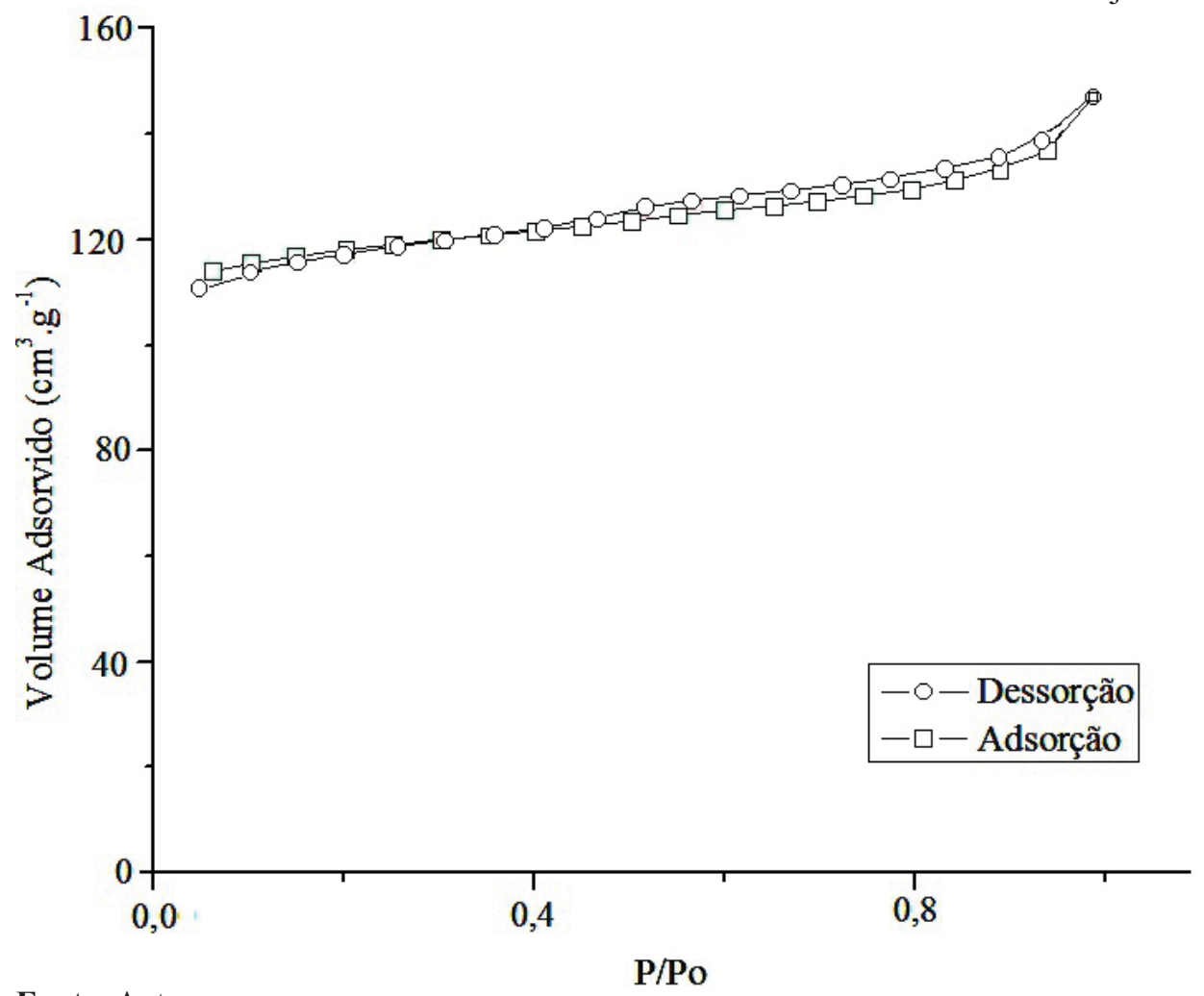

Fonte: Autor.

Figura 6 - Gráfico de isotermas de adsorção das amostras obtidos do tratamento térmico de 24 horas com a formação de Sodalita

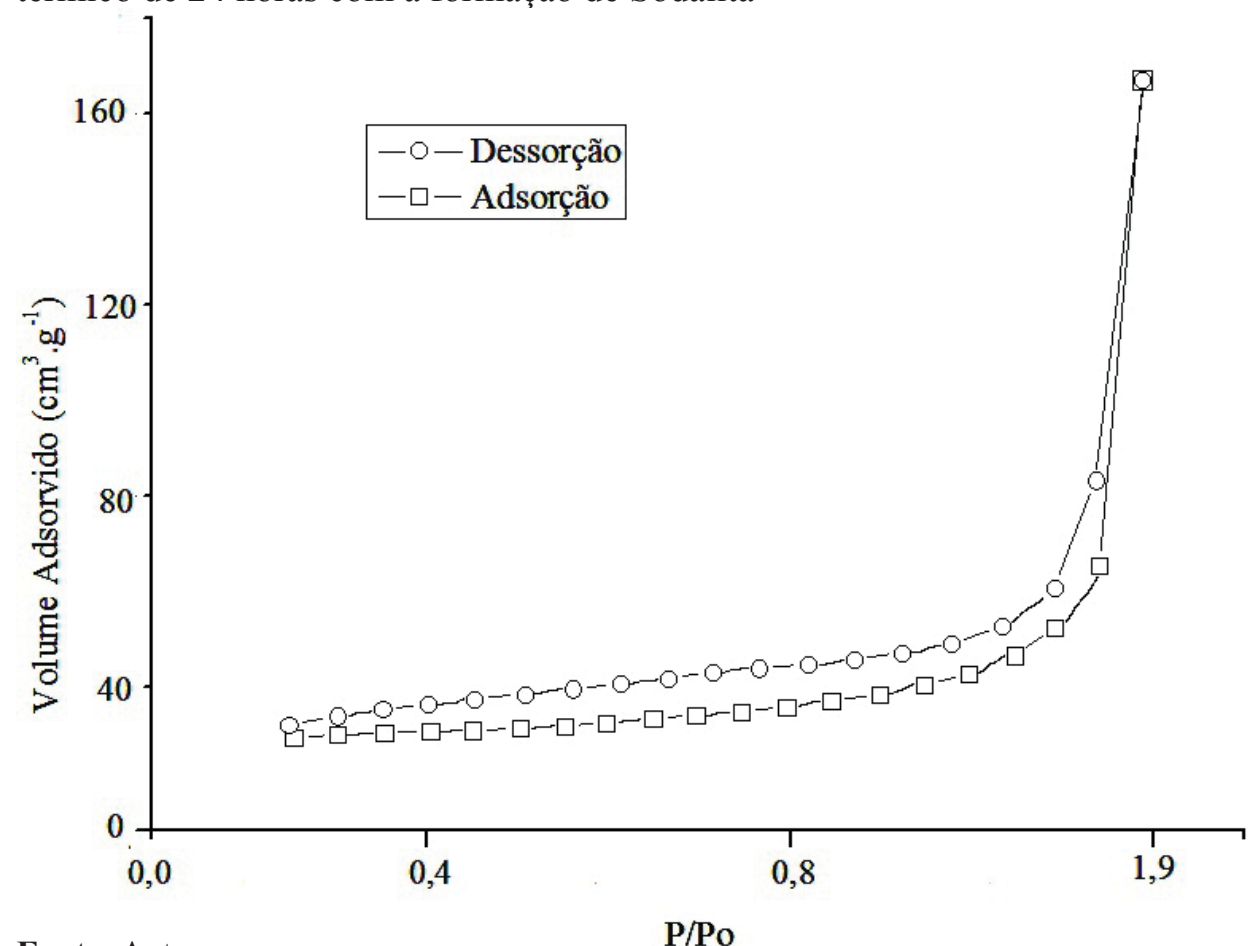

Fonte: Autor. 
A análise qualitativa de basicidade realizada utilizou a técnica de titulação de Hammet com as soluções indicadoras de amarelo de metilo, azul de timol, vermelho neutro, fenolftaleína, 4-nitroanilina e 2,4-dinitroanilina.

A análise qualitativa foi realizada uma vez que o caráter ácido ou básico do catalisador pode influir no comportamento da reação. Segundo Costa (2011), a catálise básica apresenta maior eficiência na conversão de triglicerídeo em relação à catálise ácida, além de necessitar menores quantidades de catalisador, relações de álcool/óleo e tempo de conversão. Entretanto, a catálise ácida beneficia o uso de gorduras e óleos com elevado teor de ácidos graxos livres ao contrário da catálise básica.

De acordo com os resultados obtidos na Tabela 2, as colorações das soluções indicadoras quando em contato com as zeólitas produzidas nos tratamentos térmicos de 4 e 24 horas, indicaram o caráter básico das amostras.

A Tabela 2 mostra as soluções indicadoras e os resultados obtidos na análise qualitativa de basicidade e acidez para as zeólitas produzidas no tratamento térmico de 4 e 24 horas.

A análise quantitativa de basicidade utilizou uma solução de ácido benzóico de $0,01 \mathrm{~mol} \mathrm{~L}^{-1}$ em metanol, a técnica de titulação de Hammet e soluções indicadoras representados pelos intervalos: $\mathrm{pK}_{\mathrm{BH}}=3,3$ (amarelo de metilo), $\mathrm{pK}_{\mathrm{BH}}=6,8$ (vermelho neutro), $\mathrm{pK}_{\mathrm{BH}}=8,8$ (azul de timol), $\mathrm{pK}_{\mathrm{BH}}=9,8$ (fenolftaleína), $\mathrm{pK}_{\mathrm{BH}}=15$ (2,4-dinitroanilina) e $\mathrm{pK}_{\mathrm{BH}}=18,4$ (4-nitroanilina) (JANKULOVSKA; SPIREVSKA; DIMOVA, 2011).

A análise mostrou que na Sodalita sintetizada com tratamento térmico de 24 horas, para os indicadores de Hammet que possuem um valor muito baixo ou alto de $\mathrm{pK}_{\mathrm{BH}}$ (amarelo de metilo, 2,4-dinitroanilina e 4-nitroanilina), não são detectados sítios básicos, indicando que possivelmente essa zeólita não possui sítios ativos com força muito elevada. Os sítios básicos totais calculados para esse material zeolítico foi de 0,277 mmol g-1 (Tabela 3).

A Tabela 3 mostra os intervalos de $\mathrm{pK}_{\mathrm{BH}}$ das soluções indicadoras, de sítios básicos e dos sítios totais na amostra de Sodalita sintetizada em tratamento térmico de 24 horas.

Tabela 2 - Resultados da análise de basicidade e acidez dos materiais zeolíticos obtidos no tratamento térmico de 4 e 24 horas para diferentes soluções indicadoras.

\begin{tabular}{|c|c|c|}
\hline $\begin{array}{c}\text { Solução } \\
\text { indicadora de }\end{array}$ & $\begin{array}{c}\text { Zeólita } \\
\text { semelhante à } \\
\text { Faujasita }\end{array}$ & Sodalita \\
\hline & \multicolumn{2}{|c|}{ Resultados } \\
\hline Azul de timol & Azul marinho & Azul marinho \\
\hline Fenolftaleína & Rosa carmim & Rosa carmim \\
\hline $\begin{array}{l}\text { Vermelho } \\
\text { neutro }\end{array}$ & $\begin{array}{l}\text { Marrom } \\
\text { amarelado }\end{array}$ & $\begin{array}{r}\text { Marrom } \\
\text { amarelado }\end{array}$ \\
\hline $\begin{array}{l}\text { Amarelo de } \\
\text { metilo }\end{array}$ & Amarelo & Amarelo \\
\hline $\begin{array}{c}2,4- \\
\text { Dinitroanilina }\end{array}$ & Amarelo & Amarelo \\
\hline 4-Nitroanilina & Amarelo & Amarelo \\
\hline
\end{tabular}

Fonte: Autor.

Tabela 3 - Resultados da análise quantitativa de basicidade da zeólita Sodalita sintetizada no tratamento térmico de 24 horas

\begin{tabular}{l|c|c}
\hline \multicolumn{3}{c}{ Basicidade $\left(\mathrm{mmol} \mathrm{g}^{-1}\right)$} \\
\hline Amarelo de Metilo & $\mathrm{pK}_{\mathrm{BH}}$ & Sodalita \\
Vermelho Neutro & 6,3 & - \\
Azul de Timol & 8,8 & 0,205 \\
Fenolftaleína & 9,8 & 0,036 \\
2,4-Dinitroanilina & 15 & - \\
4-Nitroanilina & 18,4 & - \\
Totais & - & 0,277 \\
\hline
\end{tabular}

Fonte: Autor. 


\section{Considerações Finais}

Com o tratamento térmico de 4 horas obtevese uma zeólita com cela unitária semelhante à estrutura cristalina da Faujasita de caráter básico e com valores de área superficial, volume de poros superior ao relatado na literatura, assim como a presença de material amorfo, detectado por difração de raios $\mathrm{X}$ entre o intervalo de $20^{\circ}-40^{\circ}$ em relação a $2 \theta$.

Para a metodologia com tratamento de 8 horas e 12 horas foi possível acompanhar a passagem da fase da zeólita com estrutura cristalina semelhante à Faujasita para Sodalita.

Os materiais zeolíticos produzidos nos tratamentos térmicos de 24 e 30 horas, apresentaram área superficial inferiores ao relatado na literatura, em que se variava a porcentagem de Sodalita básica e Hidrossodalita no material zeolítico.

Para a Sodalita produzida no tratamento de 24 horas obteve-se uma zeólita de caráter básico com sítios ativos totais de $0,277 \mathrm{mmol} \mathrm{g}^{-1}$. A falta de detecção de alguns sítios básicos (valor muito baixo ou alto de $\mathrm{pK}_{\mathrm{BH}}$ ) foram atribuídos possivelmente a ausência de sítios ativos com elevada força (JANKULOVSKA; SPIREVSKA; DIMOVA, 2011). Entretanto, estudos de Martins e Mota (2014) mostrou que mesmo catalisadores com sítios ativos baixos podem apresentar rendimentos significativos no processamento de produtos.

Para uma possível utilização destas zeólitas em reações de transesterificação de óleo vegetais, a presença destas fases secundárias, como $\mathrm{SiO}_{2}$ e material amorfo, podem reduzir a eficiência catalítica da reação, sendo necessário aumentar a relação catalisador:óleo para se obter um rendimento adequado. A presença de altos teores de hidrossodalita (sodalita hidratada) pode favorecer reações como saponificação e emulsificação. Entretanto, isto não descarta a possibilidade de utilização destas zeólitas como catalisador ou mesmo para a clarificação de óleos vegetais.

\section{Agradecimentos}

Os autores agradecem a CAPES (Coordenação de Aperfeiçoamento de Pessoal de Nível Superior), CNPq e a Fundação Araucária, pelo suporte financeiro, as empresas NALCO (Pomerode - SC) e UNAPROSIL (Rio de Janeiro - RJ) pela doação de aluminato de sódio e sílica coloidal respectivamente, ao C-LABMU (Complexo de Laboratório Multiusuários da UEPG) e ao Laboratório de Catálise e Produção de Biocombustíveis (LabCatProBio) da Universidade Federal do Paraná, Campus Palotina, pela infraestrutura e ensaios realizados.

\section{Referências}

AGUIAR, M. R. M. P.; NOVAES, A. C. Remoção de metais pesados de efluentes industriais por aluminossilicatos. Química Nova, São Paulo, v. 25, n. 6, p. 1145-1154, 2002.

ALMEIDA, K. A.; MARTINS, L.; CARDOSO, D. Preparação e propriedades de zeólitas Faujasita contendo cátions amônio. Química Nova, São Paulo, v. 33, n. 5, p. 1077-1081, 2010.

AYODELE, O. B.; ABBAS, H. F.; DAUD, W. A. W. Catalytic upgrading of oleic acid into biofuel using Mo modified zeolite supported Ni oxalate catalyst functionalized with fluoride ion. Energy Conversion and Management, Amsterdã, v. 88, n. 1, p. 1111-1119, 2014.

BALKUS, K. J.; LY, K. T. The preparation and characterization of an X-type zeolite: an experiment in solid-state chemistry. Journal of Chemical Education, Washington, v. 68, n. 10, p. 875-877, 1991.

BRAGA, A. A. C; MORGON, N. H. Descrições estruturais cristalinas de zeólitos. Química Nova, São Paulo, v. 30, n. 1, p. 178-188, 2007.

BRECK, D. W. Zeolite molecular sieves: structure, chemistry and use. London, UK: John Wiley and Sons, 1974.

CAOVILLA, M.; SMANIOTTO, A.; DALLAGO, R.; PERGHER, S. B. C. Zeólita NaX para remoção de corantes azul de metileno. Espaço Química, Rio Grande do Sul, v. 1, n. 1, p. 6-8, 2004. 
COSTA, P. P. K. G. Catalisadores químicos utilizados na síntese de biodiesel. Embrapa Agroenergia, Brasília, v. 1, n. 1, p. 25, 2011.

DALAI, A. K.; PRADHAN, N. C.; RAO, M. S.; GOKHALE, K. V. G. K. Synthesis and characterization of $\mathrm{NaX}$ and $\mathrm{Cu}$-exchanged $\mathrm{NaX}$ zeolites from silica obtained from rice husk ash. Indian Journal of Engineering \& Material Sciences, Nova Déli, v. 12, n. 3, p. 227-234, 2005.

FERNANDES-MACHADO, N. R. C.; MIOTTOBIGATÃO, D. M. M. Utilização de zeólitas a partir de xisto retortado na remoção de arsênio em águas contaminadas. Química Nova, São Paulo, v. 30, n. 5, p. 1108-1114, 2007.

FRANUS, W.; WDOWIN, M.; FRANUS, M. Synthesis and characterization of zeolites prepared from industrial fly ash. Environmental Monitoring and Assessment, Alemanha, v. 186, n. 9, p. 5721-5729, 2014.

FUNGARO, D. A.; IZIDORO, J. C. Remediação de drenagem ácida de mina usando zeólitas sintetizadas a partir de cinzas leves de carvão. Química Nova, São Paulo, v. 29, n. 4, p. 735-740, 2006.

FUNGARO, D. A.; IZIDORO, J. C.; BRUNO, M. Aplicação de material zeolítico sintetizado de cinzas de carvão como adsorvente de poluentes em água. Eclética Química, São Paulo, v. 34, n. 1, p. 45-50, 2009.

GEORGIEV, D.; BOGDANOV, B.; MARKOVSKA, I.; HRISTOV, Y. A study on the synthesis and structure of zeolite NaX. Journal of the University of Chemical Technology and Metallurgy, Bulgária, v. 48, n. 2, p. 168173, 2013.

GRECCO, S. T. F.; RANGEL, M. C.; GONZALEZ, E. U. A. Zeólitas hierarquicamente estruturadas. Química Nova, São Paulo, v. 36, n. 1, p. 131-142, 2013.

HILDEBRANDO, E. A.; ANGÉLICA, R. S.; NEVES, R. F.; VALENZUELA-DIAZ, F. R. Síntese de zeólita do tipo Faujasita a partir de um rejeito de caulim. Cerâmica, São Paulo, v. 58, n. 348, p. 453-458, 2012.

HILL, R.; HOWARD, C. Quantitative phase analysis from neutron powder diffraction data using the Rietveld method. Journal of Applied Crystallography, Chester, v. 20, n. 6, p. 467-474, 1987.
HIYOSHI, N. Nanocrystalline sodalite: preparation and application to epoxidation of 2- cyclohexen-1one with hydrogen peroxide. Applied Catalysis A: General, Amsterdã, v. 419-420, n. 1, p. 164-169, 2012.

INTERNATIONAL ZEOLITE ASSOCIATION - IZA. Synthesis recipes in the 2 nd edition of verified syntheses of zeolitic materials. Disponível em: <http://www.iza-online. org/synthesis/default.htm>. Acesso em: 2 jan. 2015.

JANKULOVSKA, M.; SPIREVSKA, I.; DIMOVA, V. A UV study of the behavior of some benzaldehyde hydrazones in acid medium. Journal of the Iranian Chemical Society. Irã, v. 8, n. 2, p. 502-512, 2011.

KUZNICKI, S. M.; LANGER, T. W.; CURRAN, J.; BELL, V. A. Macroporous aluminosilicate decant, preparation from sodalite and use in enthalpy energy wheels. United States Patent n. 6,379,436 B1, s/v, s/n, 2002. Disponível em: <http://patentimages.storage. googleapis.com/pdfs/US6379436.pdf > . Acesso em: 2 jan. 2015.

LARSON, A. C.; VON DREELE, R. B. General Structure Analysis System (GSAS). Los Alamos National Laboratory Report LAUR, v. 86, p. 748, 2000.

LOIOLA, A. R.; ANDRADE, J. C. R. A.; SASAKI, J. M.; SILVA, L. R. D.; NASSAR, E. J. Caracterização termogravimétrica e espectroscopia das propriedades ácidas da zeólita ZSM-22. Cerâmica, São Paulo, v. 56, n. 339, p. 250-254, 2010.

LUNA, F. J.; SCHUCHARDT, U. Modificação de zeólitas para uso em catálise. Química Nova, São Paulo, v. 24, n. 6, p. 885-892, 2001.

MA, F. J.; LIU, S. X.; SUN, C. Y.; LIANG, D. D.; REN, G. J.; WEI, F.; CHEN, Y. G.; SU, Z. M. A sodalite-type porous metal-organic framework with polyoxometalate templates: adsorption and decomposition of dimethyl methylphosphonate. Jounal of the American Chemical Society, Estados Unidos, v. 133, n. 12, p. 4178-4181, 2011.

MARTINS, C. X.; MOTA, C. J. A. Estudos sobre conversão direta de etanol a óxido de etileno sobre catalisadores zeolíticos. Revista Virtual de Química, São Paulo, v. 6, n. 5, p. 1282-1294, 2014. 
MELO, C. R.; RIELLA, H. G. Síntese de zeólita tipo $\mathrm{NaA}$ a partir de caulim para obtenção de zeólita $5 \mathrm{~A}$ através de troca iônica. Cerâmica, São Paulo, v. 56, n. 340, p. 340-346, 2010.

MIOTTO, D. M. M.; FERNANDES-MACHADO, N. R. C. Utilização de zeólitas modificadas CaX e MgX como adsorventes de compostos orgânicos. Acta Scientiarum:Technology, Maringá, v. 24, n. 6, p. 1627-1635, 2002.

OJHA, K.; PRADHAN, N. C.; SAMANTA, A. N. Zeolite from fly ash: synthesis and characterization. Bulletin of Material Science, Bangalore, v. 27, n. 6, p. 555-564, 2004.

PAZ, S. P. A.; ANGÉLICA, R. S.; NEVES, R. F. Síntese hidrotermal de Sodalita básica a partir de um rejeito de caulim termicamente ativado. Química Nova, São Paulo, v. 33, n. 3, p. 579-583, 2010.

PLOTEGHER, F.; RIBEIRO, C. Síntese e caracterização da zeólita ZSM-5 para uso em sistemas de adsorção química. Embrapa Pecuária Sudeste, São Carlos, v. 1, n. 107, p. 1-5, 2009.

POOLE, C.; PRIJATAMA, H; RICE, N. M. Synthesis of Zeolite adsorbents by hydrothermal treatment of PFA wastes: a comparative study. Minerals Engineering, v. 13, n. 8-9, p. 831-842, 2000.

SANTANA, D. L.; SARAIVA, A. C. F.; NEVES, R. F.; SILVA, D. L. Zeólita A sintetizada a partir de rejeitos do processo de beneficiamento de caulim. Cerâmica, São Paulo, v. 58, n. 346, p. 238-243, 2012.

SANTOS, C. G. M.; LANA, P. M. P. B.; SILVA, A. A. S.; BARBATO, C. N. Síntese da fase Sodalita com o uso do caulim da região Borborema-Seridó (RN/ PB) ativado termicamente. Holos, Rio Grande do Norte, v. 5, n. 28, p. 52-59, 2012.

SCHECHTER, W. H.; SHAKELY, R. H. Preparation of sodium superoxide. In: Handling and uses of the alkali metals. Washington DC: American Chemical Society, 1957, Vol. 19, Cap. 13, p. 124-128.

SHANBHAG, G. V.; CHOI, M.; KIM, J.; RYOO, R. Mesoporous sodalite: a novel, stable solid catalyst for base-catalyzed organic transformations. Journal of Catalysis, Nova Iorque, v. 264, n. 88, p. 88-92, 2009.
SILVESTRE, A. H. O.; VIEIRA, E. B.; BARRETO, L. S. Importância das zeólitas na indústria do petróleo e no craqueamento em leito fluidizado (fcc). Bolsista de Valor: Revista de divulgação do Projeto Universidade Petrobras e IF Fluminense, Rio de Janeiro, v. 2, n. 1, p. 63-75, 2012.

SIMÕES, A. N.; NEIVA, L. S.; RODRIGUES, M. G.; GAMA, L. Influência da temperatura na síntese hidrotérmica da zeólita Y obtida por microoondas. Cerâmica, São Paulo, v. 58, n. 348, p. 444-447, 2012.

SMITH, P.; WINGATE, C.; SILVA, L. Mobility of included sodan in sodalite. In: VIII Proceeding of the International Alumina Quality Workshop, Darwin, Austrália, 2008, p. 27-30.

STEPHENS, P. W. Phenomenological model of anisotropic peak broadening in powder diffraction. Journal of Applied Crystallography, Chester, v. 32, n. 2, p. 281-289, 1999.

TOBY, B. H. EXPGUI, a graphical user interface for GSAS. Journal of Applied Crystallography, Chester, v. 34, n. 2 , p. $210-213,2001$.

TOSHEVA, L.; VALYECHEV, V. P. Nanozeolites: synthesis, crystallizations mechanism and applications. Chemistry of Materials, Estados Unidos, v. 17, n. 10, p. 2494-2513, 2005.

VERMEIREN, W.; GILSON, J. P. Impact of zeolites on the petroleum and petrochemical industry. Topics in Catalysis, Nova Iorque, v. 52, n. 9, p. 1131-1161, 2009.

WOOLARD, C. D.; STRONG, J.; ERASMUS, C. R. Evaluation of the use of modified coal ash as potential sorbent for organic waste streams. Applied Geochemistry, Amsterdã, v. 17, n. 8, p. 1159-1164, 2002.

ZHANG, R.; CUI, H.; YANG, X.; TANG, H.; LIU, H.; LI, Y. Facile hydrothermal synthesis and photocatalytic activity of rod-like nanosized silver tungstate. Micro \& Nano Letters, Reino Unido, v. 7, n. 12, p. 1285-1288, 2012.

Recebido em 23 Janeiro, 2015 - Received on January 23, 2015 Aceito em 13 Julho, 2015 - Accepted on July 13, 2015 